\title{
Circ 0006282/miR-155 reduced inflammation in diabetic nephropathy via expression of SIRT1/NLRP3 signaling pathway
}

\author{
Dan WANG ${ }^{1 *}\left(\mathbb{D}\right.$, Zikun $Z H A N G^{1}$, Zekun $\mathrm{SI}^{1}$, Ling WANG ${ }^{1}$
}

\begin{abstract}
This study aimed to explore the effects and mechanism of circ 0006282 in Diabetic nephropathy (DN) in vitro and in vivo. 6-week-old male db/db mice were induced by intraperitoneal (I.P) injection of STZ (50 mg/kg, S0130, Sigma, St. Louis, MO, USA) for five consecutive days. HK-2 cells was incubated with high glucose for vitro model. Western blotting, immunostaining, and biochemical assays were used to obtain that inflammatory factor and protein expressions. The current study demonstrated that the serum circ 0006282 expression were reduced, and serum miR-155 expression in patients with DN were increased. Circ 0006282 reduced inflammation in kidney cell by high glucose. MiR-155 promoted inflammation in kidney cell by high glucose. Circ 0006282 reduced inflammation via activation of SIRT1/NLRP3 signaling pathway by suppression of miR-155 expression. MiR-155 regulated the anti-inflammation effects of circ 0006282 on inflammation in kidney cell by high glucose by SIRT1/NLRP 3 signaling pathway. Taken together, these results suggest that circ 0006282 may represent promising strategy for the therapy of DN.
\end{abstract}

Keywords: circ 0006282; diabetic nephropathy; miR-155; SIRT1; NLRP3.

Practical Application: This study is practical application for that circ 0006282 may represent promising strategy for the therapy of diabetic nephropathy.

\section{Introduction}

The role of inflammatory response in the pathogenesis of diabetes has recently attracted widespread attention (Du et al., 2016). Studies have reported that diabetes nephropathy (DN) is an important microvascular complication of diabetes, which has become one of the important death causes of patients with end-stage renal disease (ESRD) and diabetes (Kincaid et al., 2018; Walker et al., 2013; Vidal et al., 2020). However, to date, the comprehensive treatment by controlling blood glucose, blood pressure, blood lipids within the ideal range cannot completely prevent the occurrence and progression of DN (Sempere et al., 2017). In recent years, accumulative attention has been paid to the role of inflammatory response in the pathogenesis of diabetes. Inflammatory factors and pro-inflammatory factors have been reported to be closely associated with the occurrence and progression of $\mathrm{DN}, \mathrm{DN}$ has been considered as an inflammatory disorder. In addition, accumulative animal studies and clinical trials have proved that immune inflammatory response cells, cytokines, acute phase reactive proteins, coagulation factors, etc. play important roles in diabetic renal damage (Walker et al., 2013).

Study on DN patients have revealed that the expression of NLRP3 in renal tubular epithelial cells is significantly correlated with the level of interleukin-1 $\beta$ (IL-1 $\beta$ ), renal interstitial injury and renal function changes, indicating that the activation of NLRP3 inflammasome is involved in renal tubular oxidative stress injury of DN (Xu et al., 2018; Zhu et al., 2015; Cao et al., 2016).
With the in-depth studies on SIRT1 and other inflammatory response inhibitors in recent years, early monitoring and intervention on inflammatory response are expected in DN, thereby decreasing proteinuria (Huang et al., 2018). SIRT1 is a $\mathrm{NAD}^{+}$-dependent histone deacetylase, which is mainly distributed in mesangial cells, podocytes, renal tubular epithelial cells and renal interstitial cells in the kidney (Chen et al., 2017). Functionally, SIRT1 can regulate gene silencing, cell proliferation, differentiation, senescence, apoptosis and energy metabolism by deacetylating the receptor protein, thereby protecting kidney cells from damage and maintaining the function (He et al., 2018).

Circular RNA (circRNA) is a type of non-coding RNA (ncRNA) molecule formed by covalent binding of the 3'-terminal and the 5'-terminal after reverse splicing (Livak \& Schmittgen, 2001; Gonzalo-Calvo et al., 2015). circRNAs are prevalently distributed in multiple types of cells, which are characterized by stable structure, sequence conservation, and cell- or tissue-specific expression (Ramírez-Moya et al., 2018). The present studies have shown that circRNA can play a role through multiple patterns (Gharbi et al., 2018). To be specific, circRNA can function as a microRNA (miRNA) sponge to play its expression-regulatory role; meanwhile, it can also regulate gene transcription, cell cycle or senescence and other physiological processes through interaction with proteins (Nicoletti et al., 2014; Pearse et al., 2018).

miRNAs are a type of endogenous single-stranded smallmolecular RNA with approximately 19-25 nucleotides in length, 
which is mainly encoded by the non-coding region between the genes on the chromosome (Livak \& Schmittgen, 2001). With posttranscriptional regulatory activity, miRNAs can recognize with 3 '-untranlated region (3'-UTR) sequence of the mRNA through the principle of complementary pairing, causing degradation of the target mRNA and/or translational inhibition. miRNAs are widely distributed in humans and animals, and are associated with multiple important biological processes, including development, differentiation, proliferation and apoptosis (Wahl et al., 2016). By contrast, miRNA dysregulation is correlated with various types of diseases, such as cancer, cardiovascular disease, organ fibrosis, etc (Livak \& Schmittgen, 2001). In recent years, with the rapid development of molecular biology, gene therapy has shown broad developing prospects (Chen et al., 2018). Based on the functional characteristics of miRNAs in diseases, up-regulating or down-regulating the expression of a specific miRNA is likely to prevent and treat disease (Livak \& Schmittgen, 2001). This study aimed to explore the effects and mechanism of circ 0006282 in $\mathrm{DN}$ in vitro and in vivo.

\section{Materials and methods}

\subsection{Quantitative study of mice model}

This protocol was approved by the Ethics Committee of authhor's affiliation. Specific pathogen-free (SPF) grade 6-week-old male $\mathrm{db} / \mathrm{db}$ mice (19-20 g) were purchased from Victoria Tong Lihua (Beijing, China). The animals were housed at $23 \pm 3^{\circ} \mathrm{C}$, $55-60 \%$ humidity under a $12 \mathrm{hr}$ light/dark cycle. Mice were induced by intraperitoneal (I.P) injection of STZ $(50 \mathrm{mg} / \mathrm{kg}$, S0130, Sigma, St. Louis, MO, USA) for five consecutive days. After 12 weeks after induction of model, blood glucose levels were checked and measured blood glucose levels $(>300 \mathrm{mg} / \mathrm{dL}$ ).

\subsection{Real-time quantitative PCR (RT-qPCR)}

Total RNA was extracted from the serum or cells samples and reverse transcribed into cDNA. cDNA was PCR amplification by High Capacity cDNA Reverse Transcription Kits (Applied Biosystems, MA, USA). RT-qPCR was performed using SYBR green Master Mix (Applied Biosystems) by an ABI Prism 7500 sequence detection system (Applied Biosystems). Relative gene expression was analyzed using the $2^{-\Delta \Delta C T}$ method.

\subsection{Biochemical parameters and HE staining}

After the mice were sacrificed, serum samples were collected at $1000 \mathrm{~g}$ and used to measure creatinine using an AU680 automated chemistry analyzer (Beckman Coulter, Inc., Brea, CA, USA). Meanwhile, Kidney samples of all mice were fixed with $4 \%$ paraformaldehyde for $24 \mathrm{~h}$. Kidney samples sections were cut $5 \mu \mathrm{m}$ from paraffin-embedded kidney samples and were stained with Hematoxylin-Eosin(H-E) sassy. Images were captured using an optical microscope (Carl Zeiss, Oberkochen, Germany).

\subsection{Cell culture and transfection}

HK-2 cells were purchased from Shanghai cell bank, Chinese academy of sciences and were grown in F12/DMEM supplemented with $10 \%(v / v)$ fetal calf serum (FCS, Gibco, NY, USA) at $37^{\circ} \mathrm{C}$ in $95 \%$ humidified air and $5 \% \mathrm{CO}_{2}$. $\mathrm{HK}-2$ cells was incubated with high glucose $(40.9 \mathrm{mM})$ for $48 \mathrm{~h}$. Cell was transfected using Lipofectamine 2000 (Invitrogen, USA) with circ 0006282, si-circ 0006282, miR-155, si-miR-155 or negative mimics.

\subsection{Measurement of inflammation}

Cell was collected at $1000 \mathrm{~g}$ for induction of model and TNF- $\alpha$, IL-1 $\beta$, IL- 6 and IL- 18 levels were measured using inflammation ELISA Kits according to the methods of manufacturer instructions. Absorbance were measured using UniCel DxC 600 Chemistry Analyzer (Beckman Coulter, CA, USA).

\subsection{Microarray analysis}

Microarray experiments were performed at the Genminix Informatics (China). Gene expression was analyzed using the Human Gene Expression 4x44K v2 Microarray Kit (Agilent, Santa Clara, CA). Data were obtained using the Agilent Feature Extraction software.

\subsection{Western blotting}

Total protein was extracted with a RIPA lysis solution and protein content was measured using BCA assay. An equal amount of protein were separated using SDSPAGE and then transferred to a nitrocellulose membrane. Membrane was blocked using $5 \%$ skim milk for $2 \mathrm{~h}$ at room temperature and incubated with primary antibodies: SIRT1, NLRP 3 and GAPDH at $4{ }^{\circ} \mathrm{C}$ over-night. Membrane was incubated with peroxidase-labeled secondary antibodies at $37^{\circ} \mathrm{C}$ for $1 \mathrm{~h}$. The protein bands were observed using ECL assay and quantified with the ChemiDoc XRS + imaging system (Bio-Rad, Hercules, CA, USA).

\subsection{Statistical analysis}

Results are expressed as means \pm SEM. Student's t-test was used to analyze the difference between two independent groups. One-way analysis of variance (ANOVA) and Tukey's post test was used to analyze the difference between two independent groups. $\mathrm{p}$ values $<0.05$ were considered to indicate statistical significance.

\section{Results}

\subsection{Circ 0006282 and miR-155 expression in DN mice}

To study the function of circRNAs and microRNAs in mice with DN, the changes of Circ 0006282 and miR-155 expression in patients with DN were measured using RT-qPCR. As showed in Figure 1A, 1B, the serum circ 0006282 expression in patients with $\mathrm{DN}$ were reduced, and serum miR-155 expression in patients with DN were increased. In the same study a significant negative correlation was found between circ 0006282 and miR-155 (Figure 1C). There were a significant positive correlation was found between miR-155 and creatinine, and a significant negative correlation was found between circ 0006282 and creatinine (Figure 1D, 1E). Luciferase reporter activity in miRNA-155 group was lower than that of negative group (Figure 1F). Figure $1 \mathrm{H}$ showed that circ 0006282 binding site miRNA-155 (Figure 1G). Over-expression of 

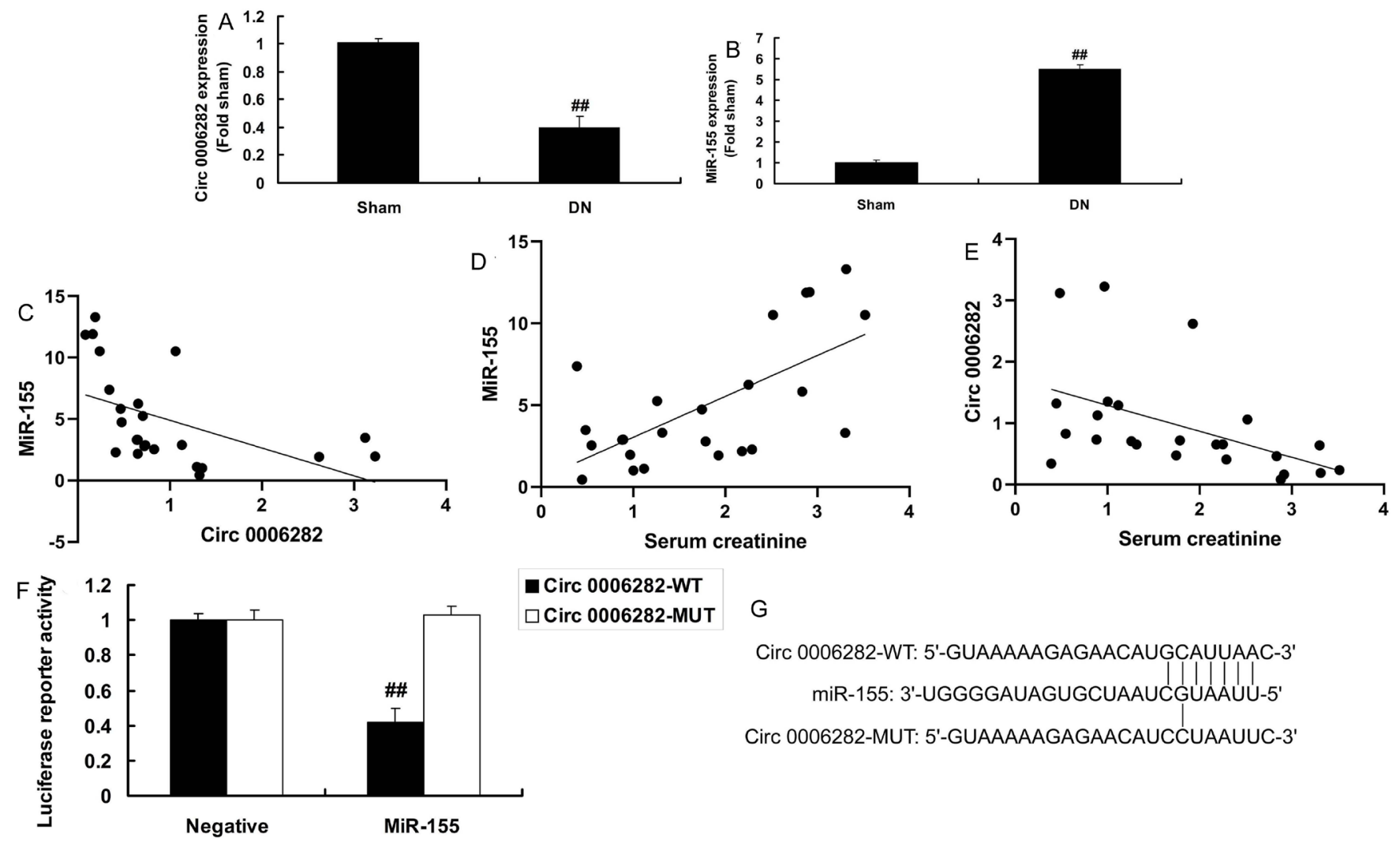

口 Circ 0006282-WT $\square$ Circ 0006282-MUT G
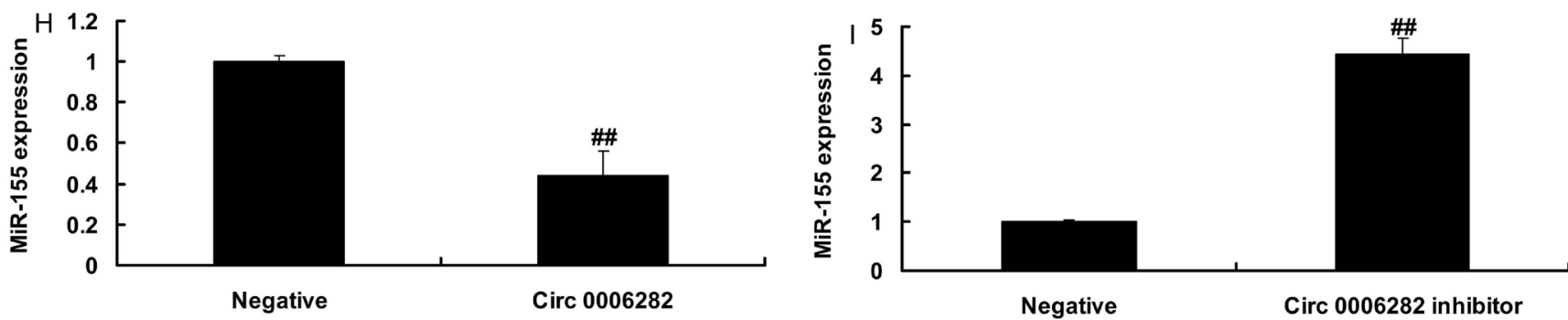

Figure 1. Circ 0006282 and miR-155 expression in DN mice. Circ 0006282 and miR-155 expression in DN mice (A and B), between circ 0006282 and miR-155 (C), between miR-155 and creatinine (D), between circ 0006282 and creatinine (E); luciferase reporter activity (F), circ 0006282 binding site miRNA-155 (G), over-expression of circ 0006282 reduced miR-155 expression $(\mathrm{H})$, and down-regulation of circ 0006282 promoted miR-155 expression (I). Sham, normal volunteers group; DN, DN patients group; ${ }^{\# \# ~}<0.01$ compared with DN patients group; $<<0.01$ compared with DN patients group.

circ 0006282 reduced miR-155 expression, and down-regulation of circ 0006282 promoted miR-155 expression (Figure 1H, 1I).

\subsection{Circ 0006282 reduced inflammation in kidney cell by high glucose}

To confirm that the anti-inflammation effects of circ 0006282 in DN, we used HK-2 cells by high glucose for vitro model of DN. Over-expression of circ 0006282 reduced TNF- $\alpha$, IL-1 $\beta$, IL- 6 and IL-18 cell levels in kidney cell by high glucose (Figure 2A-2E). However, down-regulation of circ 0006282 reduced circ 0006282 expression, promoted TNF- $\alpha$, IL-1 $\beta$, IL- 6 and IL-18 cell levels in kidney cell by high glucose (Figure 2F-2J).

\subsection{Circ 0006282 regulated inflammation via SIRT1/NLRP3 signaling pathway by miR-155}

Gene chip analyzed the miR-155 regulated signaling pathway and we found that 23 gene were chronic inflammation of miR-155 regulation gene (Figure 3A, 3B). Volcano Plot showed that NLPR 3 and so on were up-regulated, and SIRT1 and so on were down-regulated (Figure 3C). Analysis result of Figure 3D showed that SIRT1/NLRP3 was the common point of DN regulation gene, miR- 155 regulation gene and inflammation regulation gene. Lastly, SIRT1/NLRP3 signaling pathway may be downstream channel (Figure 3E). Luciferase reporter activity in miRNA-155 group was lower than that of negative group (Figure $4 \mathrm{~A}$ ). Figure $1 \mathrm{H}$ showed that SIRT1 binding site miRNA-155 (Figure 4B). Overexpression of miR-155 suppressed SIRT1 protein expression, 


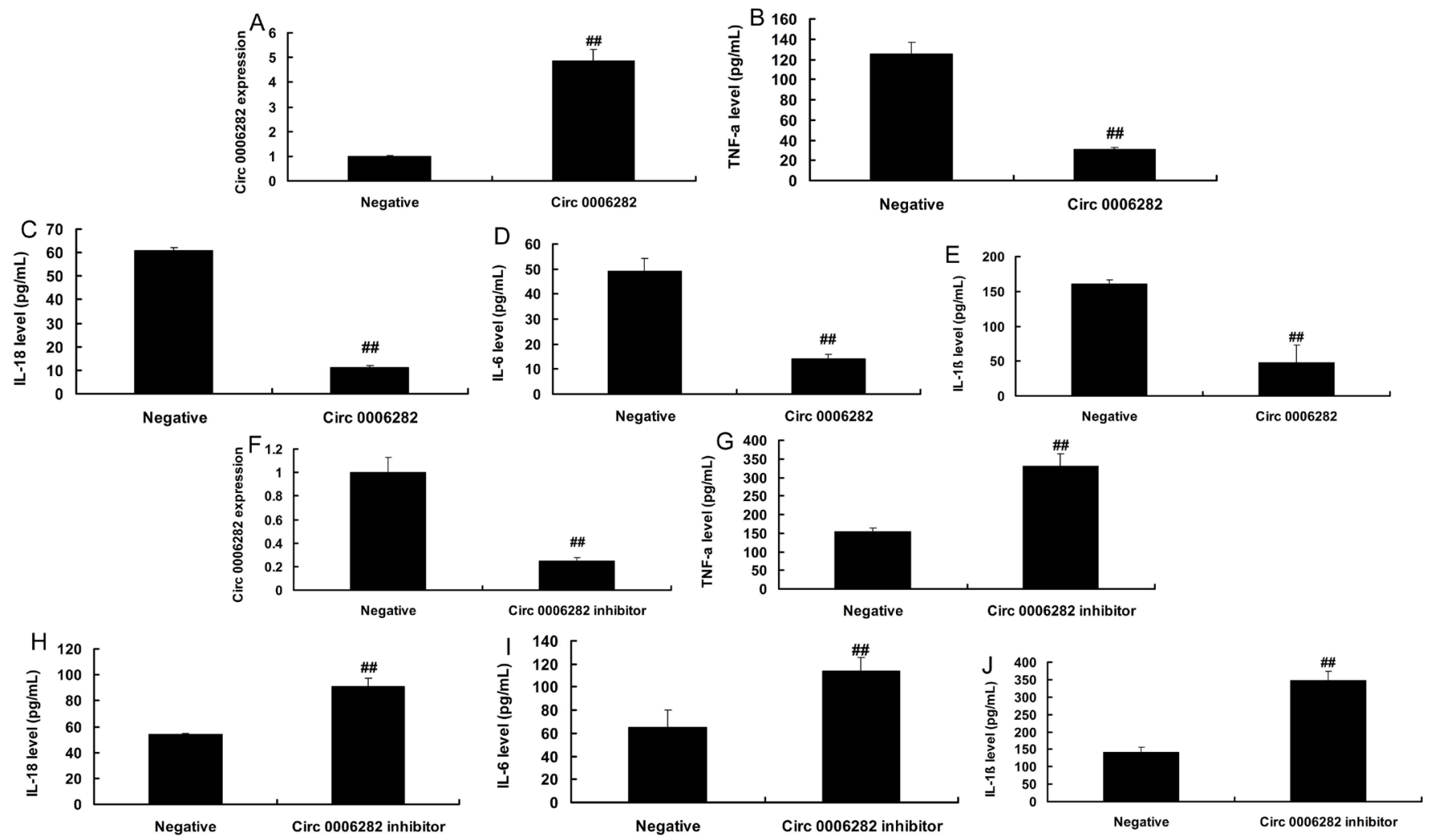

Figure 2. Circ 0006282 reduced inflammation in kidney cell by high glucose. Circ 0006282 expression (A), TNF- $\alpha$, IL-1 $\beta$, IL-6 and IL-18 cell levels (B, C, D and E) by over-expression of circ 0006282; Circ 0006282 expression (F), TNF- $\alpha$, IL-1 $\beta$, IL-6 and IL-18 cell levels (G, H, I and J) by down-rgulation of circ 0006282. Negative, negative mimics group; Circ 0006282, over-expression of circ 0006282 group; Circ 0006282 inhibitor, down-regulation of circ 0006282 group. ${ }^{\# \#} \mathrm{p}<0.01$ compared with negative mimics group.
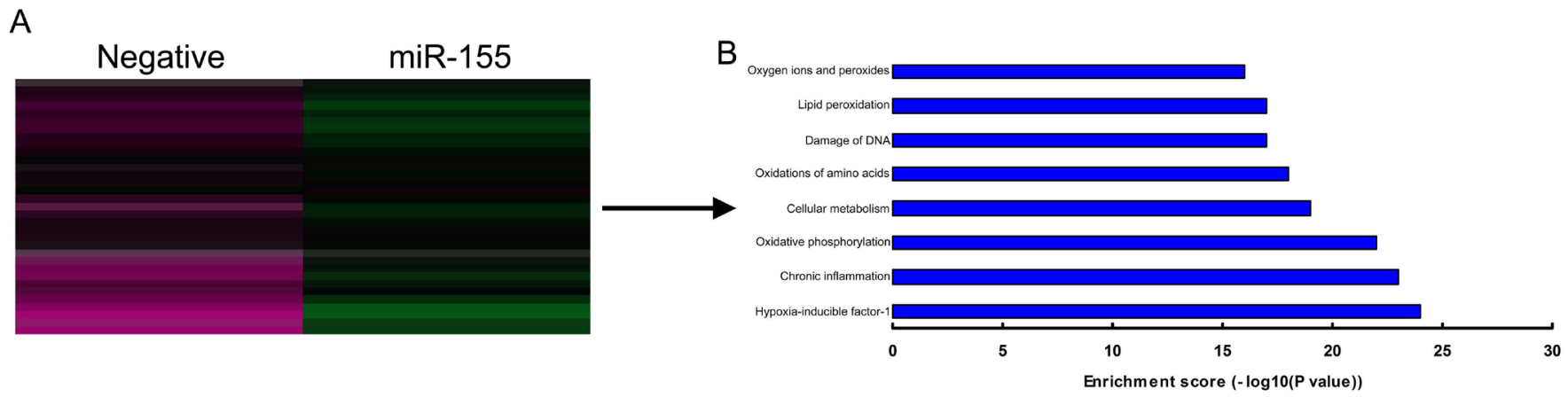

E

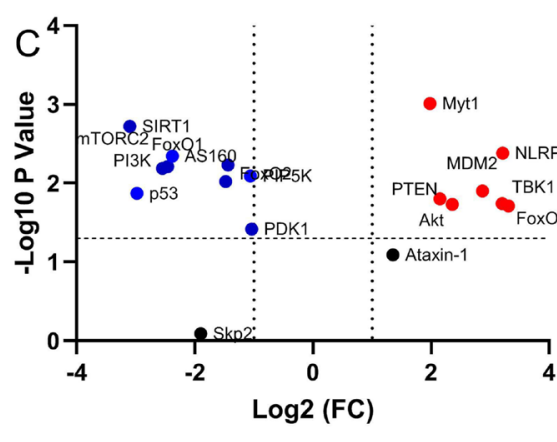

D
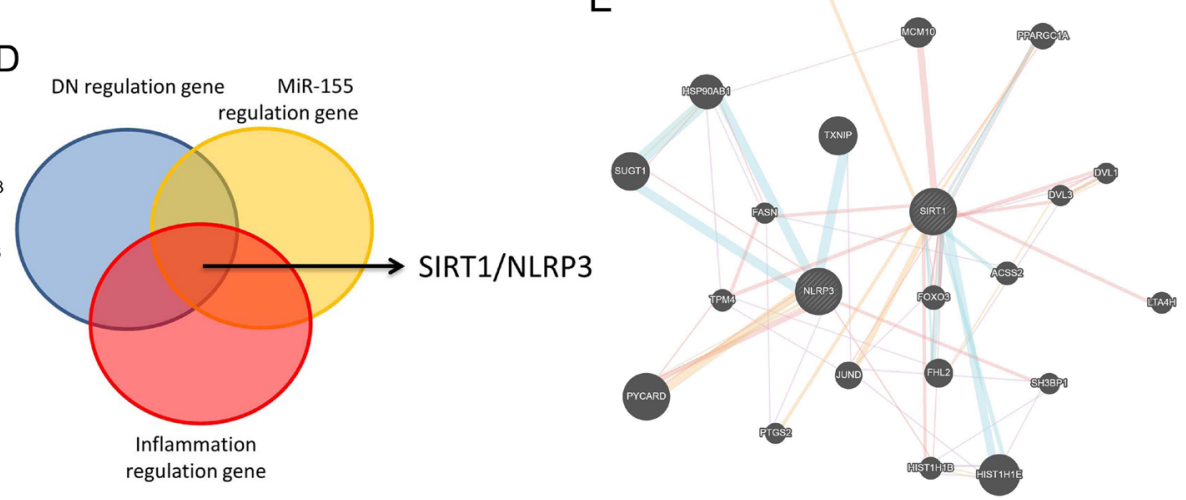

Figure 3. miR-155 regulated inflammation via SIRT1/NLRP3 signaling pathway. Heat map and analysis results of gene chip (A and B), Volcano Plot (C), analysis results (D), network of SIRT1/NLRP3 signaling pathway (E). 

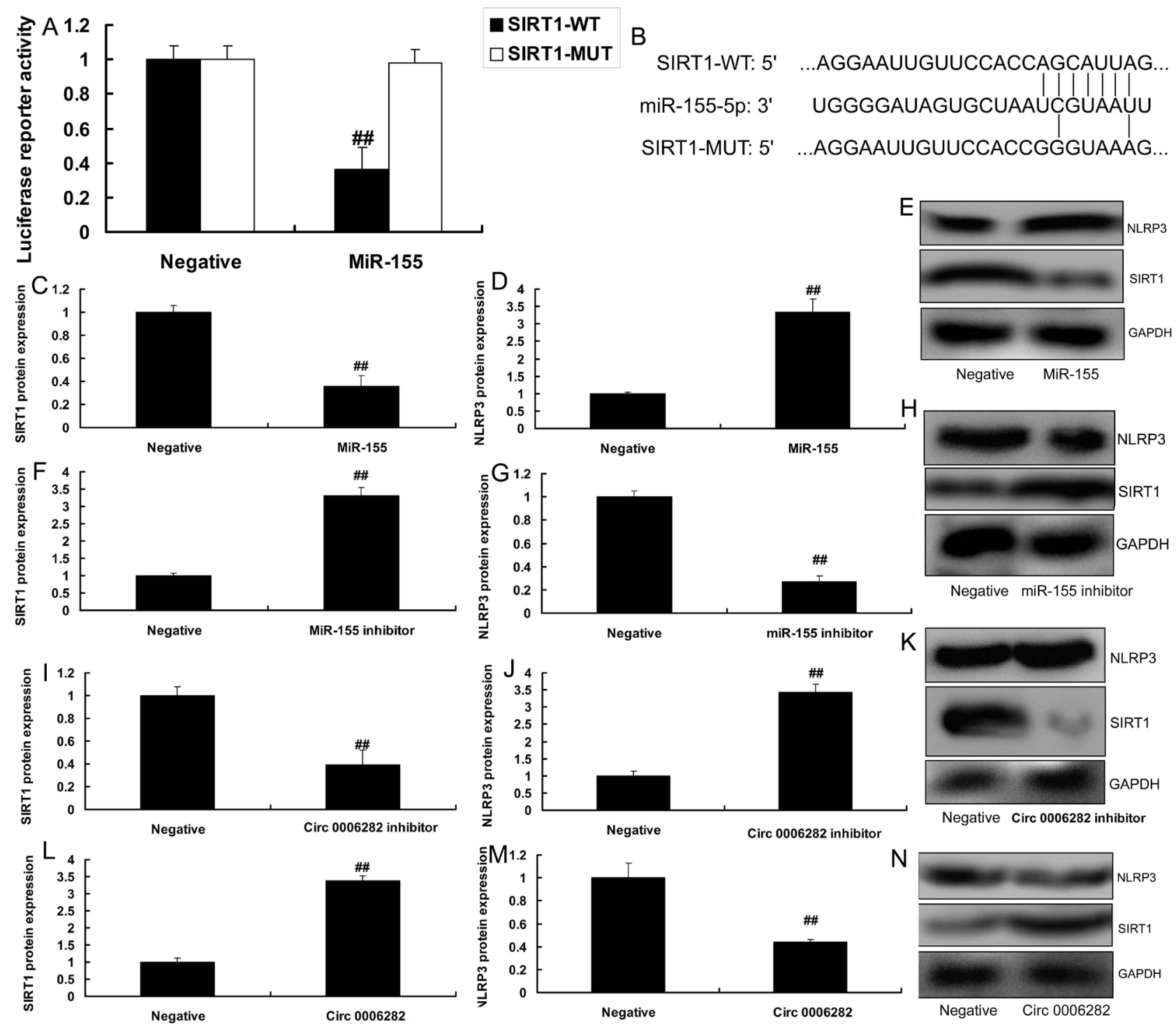

Figure 4. Circ 0006282 regulated inflammation via SIRT1/NLRP3 signaling pathway by miR-155. Luciferase reporter activity (A), SIRT1 binding site miRNA-155 (B), SIRT1/NLRP3 signaling pathway by over-expression of miR-155 (C, D and E), SIRT1/NLRP3 signaling pathway by downregulation of miR-155 (F, G and H), SIRT1/NLRP3 signaling pathway by down-regulation of Circ 0006282 (I, J and K), SIRT1/NLRP3 signaling pathway by over-expression of Circ 0006282 (L, M and N). Negative, negative mimics group; miR-155, over-expression of miR-155 group; miR155 inhibitor, down-regulation of miR-155 group; Circ 0006282, over-expression of circ 0006282 group; Circ 0006282 inhibitor, down-regulation of circ 0006282 group. ${ }^{\# \#} \mathrm{p}<0.01$ compared with negative mimics group.

and induced NLRP3 protein expression (Figure 4C-4E). Downregulation of miR-155 induced protein expression, and suppressed NLRP3 protein expression (Figure 4F-4H). Down-regulation of circ 0006282 suppressed SIRT1 protein expression, and induced NLRP3 protein expression (Figure 4I-4K). Over-expression of circ 0006282 induced protein expression, and suppressed NLRP3 protein expression (Figure 4L-4N).

\subsection{MiR-155 promoted inflammation in kidney cell by high glucose}

To confirm that the pro-inflammation effects of miR-155 in $\mathrm{DN}$, we used HK-2 cells by high glucose for vitro model of DN. Over-expression of miR-155 promoted TNF- $\alpha$, IL- $1 \beta$, IL- 6 and
IL-18 cell levels in kidney cell by high glucose (Figure 5A-5E). However, down-regulation of miR- 155 inhibited miR-155 expression, and reduced TNF- $\alpha$, IL- $1 \beta$, IL- 6 and IL- 18 cell levels in kidney cell by high glucose (Figure 5F-5J).

\subsection{MiR-155 regulated the anti-inflammation effects of circ 0006282 on inflammation in kidney cell by high glucose by SIRT1/NLRP3 signaling pathway}

To systematically identify the mechanism of circ 0006282 in $\mathrm{DN}$ and we analyzed the function of miR-155 in anti-inflammation effects of circ 0006282 in DN. MiR-155 expression was upregulation in circ 0006282 and miR-155 group, comparing 

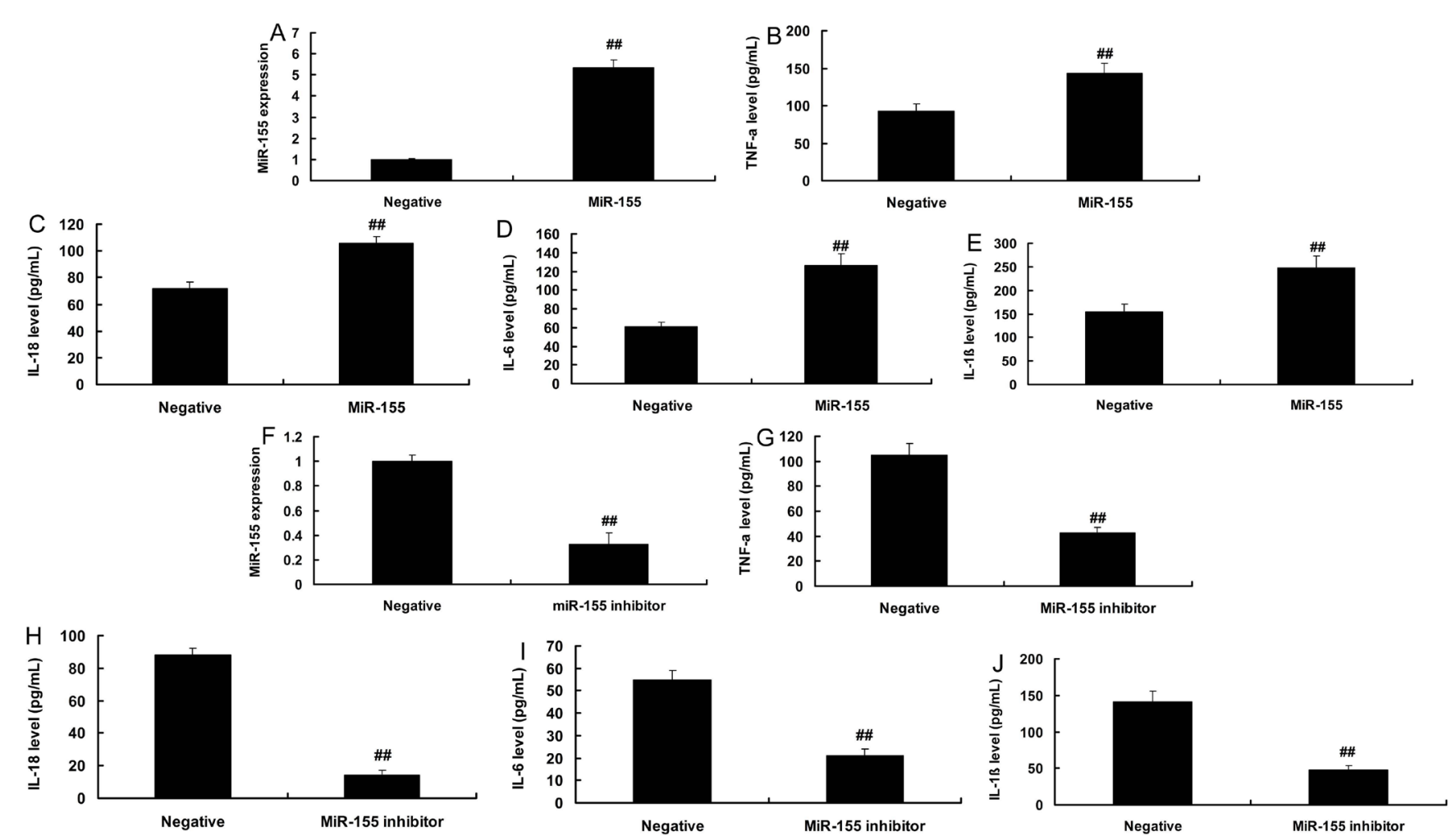

Figure 5. MiR-155 promoted inflammation in kidney cell by high glucose. MiR-155 expression (A), TNF- $\alpha$, IL-1 $\beta$, IL-6 and IL-18 cell levels (B, C, D and E) by over-expression of miR-155; miR-155 expression (F), TNF- $\alpha$, IL-1 $\beta$, IL-6 and IL-18 cell levels ( $G$, H, I and J) by down-rgulation of miR-155. Negative, negative mimics group; miR-155, over-expression of miR-155 group; miR-155 inhibitor, down-regulation of miR-155 group. ${ }^{\# \#} \mathrm{p}<0.01$ compared with negative mimics group.
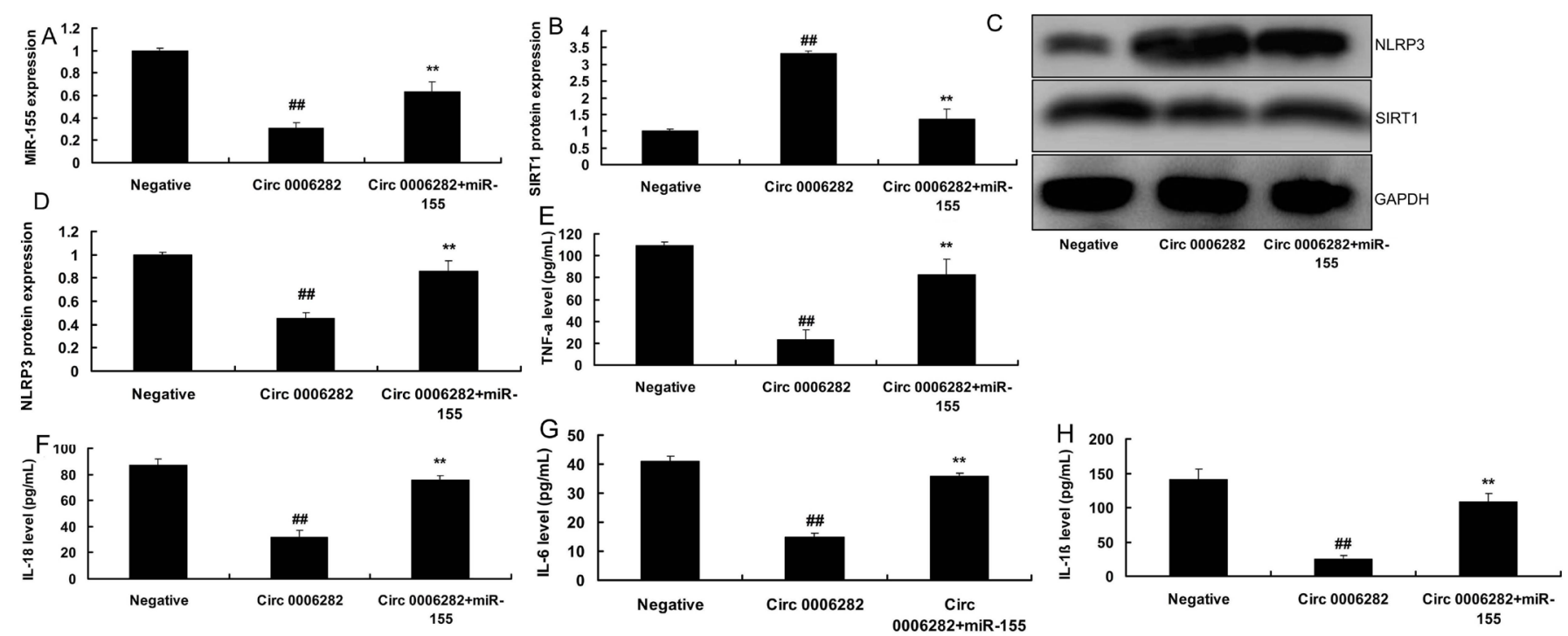

Figure 6. MiR-155 regulated the anti-inflammation effects of circ 0006282 on inflammation in kidney cell by high glucose by SIRT1/NLRP3 signaling pathway. MiR-155 expression (A), SIRT1/NLRP3 signaling pathway (B, C and D), TNF- $\alpha$, IL-1 $\beta$, IL-6 and IL-18 cell levels (E, F, G and H). Negative, negative mimics group; Circ 0006282, over-expression of circ 0006282 group; Circ $0006282+$ miR-155, over-expression of circ 0006282 and miR-155 group. ${ }^{* \#} \mathrm{p}<0.01$ compared with negative mimics group; ${ }^{* *} \mathrm{p}<0.01$ compared with over-expression of circ 0006282 group.

to only circ 0006282 group (Figure 6A). Over-expression of miR-155 reduced SIRT1 protein expression and induced NLPR3 protein expression in HK-2 cells by high glucose by circ 0006282 , comparing with only circ 0006282 group
(Figure 6B, 6C). Over-expression of miR-155 increased TNF- $\alpha$, IL-1 $\beta$, IL- 6 and IL- 18 cell levels in HK-2 cells by high glucose by circ 0006282 , comparing with only circ 0006282 group (Figure 6D-6G). 

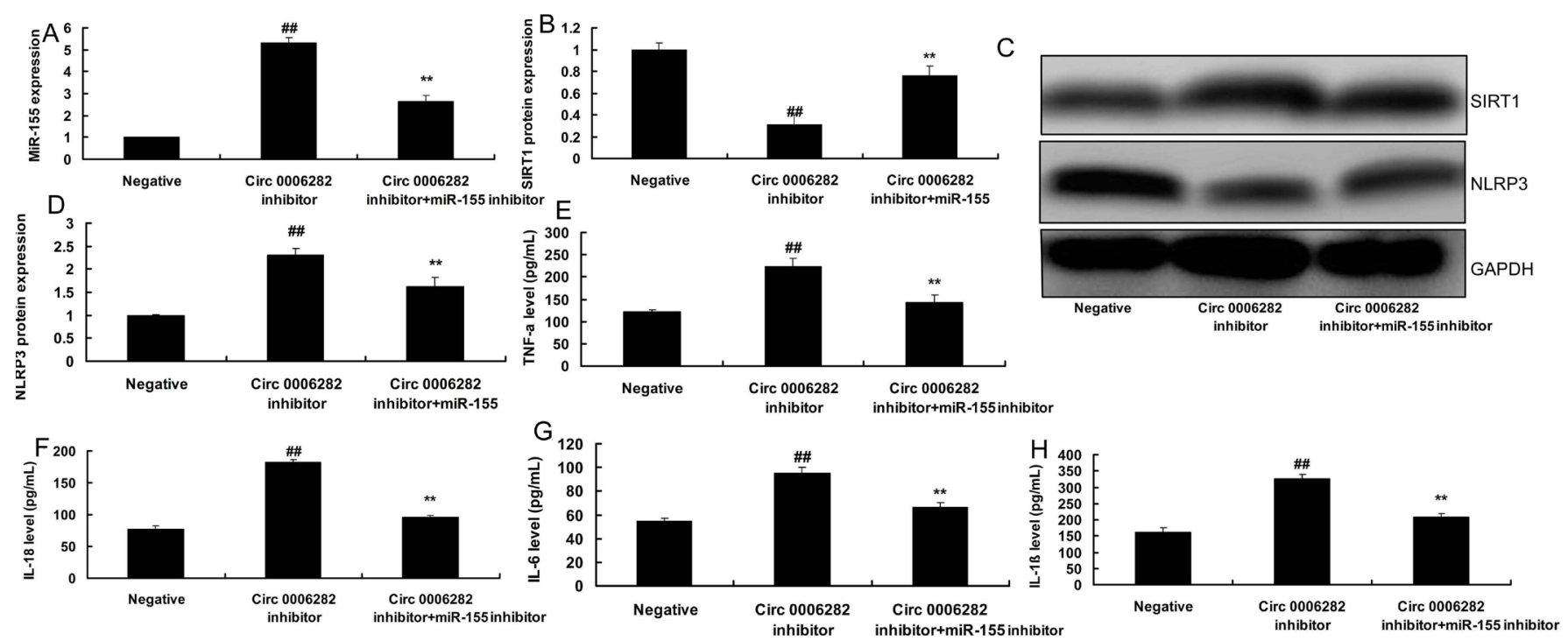

Figure 7. MiR-155 inhibitor regulated the effects of circ 0006282 inhibitor on inflammation in kidney cell by high glucose by SIRT1/NLRP3 signaling pathway.MiR-155 expression (A), SIRT1/NLRP3 signaling pathway (B, C and D), TNF- $\alpha$, IL-1 $\beta$, IL-6 and IL- 18 cell levels (E, F, G and H). Negative, negative mimics group; Circ 0006282 inhibitor, down-regulation of circ 0006282 group; Circ 0006282 inhibitor+miR-155 inhibitor, down-regulation of circ 0006282 and miR-155 group. ${ }^{\# *} \mathrm{p}<0.01$ compared with negative mimics group; ${ }^{* *} \mathrm{p}<0.01$ compared with down-regulation of circ 0006282 group.

Meanwhile, si-miR-155 reduced miR-155 expression in HK-2 cells by high glucose by si-circ 0006282 , comparing with only si-circ 0006282 group (Figure 7A). Down-regulation of miR-155 induced SIRT1 protein expression and suppressed NLPR3 protein expression in HK-2 cells by high glucose by si-circ 0006282, comparing with only si-circ 0006282 group (Figure 7B, 7C). Down-regulation of miR-155 decreased TNF- $\alpha$, IL-1 $\beta$, IL- 6 and IL-18 cell levels in HK- 2 cells by high glucose by circ 0006282 , comparing with only circ 0006282 group (Figure 7D-7G).

\section{Discussion}

DN is one of the important chronic complications of diabetic patients, which is mostly characterized by the sclerosis of diabetic glomerular cells (Wu et al., 2018). Both type 1 and type 2 diabetes have a certain probability of progressing into $\mathrm{DN}$, which would eventually lead to renal necrosis (Livak \& Schmittgen, 2001). Moreover, the life and health of diabetic patients are directly threatened by $\mathrm{DN}$ (He et al., 2017). Due to the rapid development of modern medical technology, the advent of various therapeutic approaches has greatly decreased the ketoacidosis-related death rate in diabetic patients (Maegdefessel et al., 2014). At present, the diverse complications of diabetes have become the main cause of death in diabetic patients, such as DN and cardiovascular disease caused by diabetes. DN is the most important chronic microvascular complication of diabetic patients, which is also one of the important lethal factors for diabetic patients. The inflammatory process plays an extremely important role in the occurrence and progression of DN. Additionally, DN is closely associated with its inflammatory response mechanism. The current study demonstrated that the serum circ 0006282 expression were reduced, and serum miR-155 expression in mice with DN were increased. Circ 0006282 reduced inflammation in kidney cell by high glucose. MiR-155 promoted inflammation in kidney cell by high glucose. He et al. (2020) showed that Circ 0006282 contributes to the progression and cell growth of gastric cancer by miRNA- 155 .

Inflammation is one of the important pathogenesis of DN (Li et al., 2018). A large number of inflammatory factors are involved in the pathogenesis of DN. Recent studies have found that apoptosis is also involved (Zhu et al., 2015). However, at present, studies on the involvement of NLRP3 inflammasome and apoptosis-associated speck-like protein containing a CARD (ASC) in DN have been rarely reported (Li et al., 2018). Therefore, in this study, male SD rats of the DN model were used to investigate the specific expression of NLRP3 inflammasome in the DN model and the correlation of NLRP3 with apoptosis. Our results indicate that circ 0006282 reduced inflammation via activation of SIRT1/NLRP3 signaling pathway by suppression of miR-155 expression. Li et al. (2019) reported that miR155 promotes macrophage pyroptosis by NLRP 3 inflammasome in porphyromonas gingivalis.

SIRT1, a highly-conserved deacetylase, is not only closely associated with the energy metabolism and reducing state of cells, but also involved in regulating various biological processes (Li et al., 2017). Studies have demonstrated that SIRT1 is closely correlated with the pathogenesis of $\mathrm{DN}$, which is a novel potential therapeutic target for DN (Liao et al., 2018; Wang et al., 2018). The present data showed that miR- 155 regulated the anti-inflammation effects of circ 0006282 on inflammation in kidney cell by high glucose by SIRT1/NLRP3 signaling pathway. $\mathrm{Qu}$ et al. (2020) suggest that miR-155 promoted osteoblastic differentiation by SIRT1 in high glucose model.

In summary, the current study indicates that circ 0006282 reduced inflammation via activation of SIRT1/NLRP3 signaling pathway 
by suppression of miR-155 expression. Results collected from the present study suggest that circ 0006282 may be of benefits to reduce inflammation of $\mathrm{DN}$ and thus retard pathological development of DN. Taken together, these results suggest that circ 0006282 may represent promising strategy for the therapy of DN.

\section{References}

Cao, G., Jiang, N., Hu, Y., Zhang, Y., Wang, G., Yin, M., Ma, X., Zhou, K., Qi, J., Yu, B., \& Kou, J. (2016). Ruscogenin attenuates cerebral ischemia-induced blood-brain barrier dysfunction by suppressing txnip/nlrp3 inflammasome activation and the MAPK pathway. International Journal of Molecular Sciences, 17(9), 1418. http:// dx.doi.org/10.3390/ijms17091418. PMid:27589720.

Chen, W., Zhao, W., Yang, A., Xu, A., Wang, H., Cong, M., Liu, T., Wang, P., \& You, H. (2017). Integrated analysis of microRNA and gene expression profiles reveals a functional regulatory module associated with liver fibrosis. Gene, 636, 87-95. http://dx.doi.org/10.1016/j. gene.2017.09.027. PMid:28919164.

Chen, Y., Yang, X. Q., Tseng, B. Y., Tsai, Y. H., Tseng, S. H., Lee, C. H., \& Yao, C. L. (2018). Deferoxamine preconditioning activated hypoxia-inducible factor- $1 \alpha$ and MyD88-dependent Toll-like receptor 4 signaling in intestinal stem cells. Journal of Pediatric Surgery, 53(11), 2349-2356. http://dx.doi.org/10.1016/j.jpedsurg.2018.01.023. PMid:29475626.

Du, F., Yuan, P., Zhao, Z. T., Yang, Z., Wang, T., Zhao, J. D., Luo, Y., Ma, F., Wang, J. Y., Fan, Y., Cai, R. G., Zhang, P., Li, Q., Song, Y. M., \& Xu, B. H. (2016). Erratum: a miRNA-based signature predicts development of disease recurrence in HER2 positive breast cancer after adjuvant trastuzumab-based treatment. Scientific Reports, 6(1), 35509. http://dx.doi.org/10.1038/srep35509. PMid:27739502.

Gharbi, S., Khateri, S., Soroush, M. R., Shamsara, M., Naeli, P., Najafi, A., Korsching, E., \& Mowla, S. J. (2018). MicroRNA expression in serum samples of sulfur mustard veterans as a diagnostic gateway to improve care. PLoS One, 13(3), e0194530. http://dx.doi.org/10.1371/ journal.pone.0194530. PMid:29566027.

Gonzalo-Calvo, D., Dávalos, A., Montero, A., García-González, Á., Tyshkovska, I., González-Medina, A., Soares, S. M., Martínez-Camblor, P., Casas-Agustench, P., Rabadán, M., Díaz-Martínez, Á. E., Úbeda, N., \& Iglesias-Gutiérrez, E. (2015). Circulating inflammatory miRNA signature in response to different doses of aerobic exercise. Journal of Applied Physiology, 119(2), 124-134. http://dx.doi.org/10.1152/ japplphysiol.00077.2015. PMid:25997943.

He, H., Cai, M., Zhu, J., Xiao, W., Liu, B., Shi, Y., Yang, X., Liang, X., Zheng, T., Hu, S., Jia, X., Chen, S., Wang, J., Qin, Y., \& Lai, S. (2018). miR-148a-3p promotes rabbit preadipocyte differentiation by targeting PTEN. In Vitro Cell Dev Biol Anim., 54(3), 241-249. http://dx.doi. org/10.1007/s11626-018-0232-z. PMid:29426973.

He, Y., Wang, Y., Liu, L., Liu, S., Liang, L., Chen, Y., \& Zhu, Z. (2020). Circular RNA circ_0006282 contributes to the progression of gastric cancer by sponging miR-155 to upregulate the expression of FBXO22. OncoTargets and Therapy, 13, 1001-1010. http://dx.doi.org/10.2147/ OTT.S228216. PMid:32099403.

He, Z., Zhou, Y., Lin, L., Wang, Q., Khor, S., Mao, Y., Li, J., Zhen, Z., Chen, J., Gao, Z., Wu, F., Zhang, X., Zhang, H., Xu, H. Z., Wang, Z., \& Xiao, J. (2017). Dl-3-n-butylphthalide attenuates acute inflammatory activation in rats with spinal cord injury by inhibiting microglial TLR4/NF- $\kappa B$ signalling. Journal of Cellular and Molecular Medicine, 21(11), 3010-3022. http://dx.doi.org/10.1111/jcmm.13212. PMid:28842949.
Huang, S. H., Zhang, T., Zhao, C. G., Qin, J., Qi, P., Li, F. T., \& He, X. J. (2018). Aclidinium bromide inhibits human glioma cell proliferation, migration and invasion and promotes apoptosis via the PI3K/ AKT signaling pathway. Neoplasma, 65(6), 865-871. http://dx.doi. org/10.4149/neo_2018_171117N705. PMid:29940755.

Kincaid, R. P., Panicker, N. G., Lozano, M. M., Sullivan, C. S., Dudley, J. P., \& Mustafa, F. (2018). MMTV does not encode viral microRNAs but alters the levels of cancer-associated host microRNAs. Virology, 513, 180-187. http://dx.doi.org/10.1016/j.virol.2017.09.030. PMid:29096160.

Li, C., Yin, W., Yu, N., Zhang, D., Zhao, H., Liu, J., Liu, J., Pan, Y., \& Lin, L. (2019). miR-155 promotes macrophage pyroptosis induced by Porphyromonas gingivalis through regulating the NLRP3 inflammasome. Oral Diseases, 25(8), 2030-2039. http://dx.doi. org/10.1111/odi.13198. PMid:31529565.

Li, S., Xu, T., Liu, S., Liu, Z., Pi, Z., Song, F., \& Jin, Y. (2018). Exploring the potential pharmacodynamic material basis and pharmacologic mechanism of the Fufang-Xialian-Capsule in chronic atrophic gastritis by network pharmacology approach based on the components absorbed into the blood. Royal Society Open Science, 5(6), 171806. http://dx.doi.org/10.1098/rsos.171806. PMid:30110485.

Li, Y., Ma, X., Wang, Y., \& Li, G. (2017). miR-489 inhibits proliferation, cell cycle progression and induces apoptosis of glioma cells via targeting SPIN1-mediated PI3K/AKT pathway. Biomedicine and Pharmacotherapy, 93, 435-443. http://dx.doi.org/10.1016/j. biopha.2017.06.058. PMid:28666210.

Liao, Y. X., Zhang, Z. P., Zhao, J., \& Liu, J. P. (2018). Effects of fibronectin 1 on cell proliferation, senescence and apoptosis of human glioma cells through the PI3K/AKT signaling pathway. Cellular Physiology and Biochemistry, 48(3), 1382-1396. http://dx.doi.org/10.1159/000492096.

Livak, K. J., \& Schmittgen, T. D. (2001). Analysis of relative gene expression data using real-time quantitative PCR and the $2-\triangle \Delta \mathrm{CT}$ method. Methods, 25(4), 402-408. http://dx.doi.org/10.1006/ meth.2001.1262. PMid:11846609.

Maegdefessel, L., Spin, J. M., Raaz, U., Eken, S. M., Toh, R., Azuma, J., Adam, M., Nagakami, F., Heymann, H. M., Chernugobova, E., Jin, H., Roy, J., Hultgren, R., Caidahl, K., Schrepfer, S., Hamsten, A., Eriksson, P., McConnell, M. V., Dalman, R. L., \& Tsao, P. S. (2014). miR-24 limits aortic vascular inflammation and murine abdominal aneurysm development. Nature Communications, 5(1), 5214. http:// dx.doi.org/10.1038/ncomms6214. PMid:25358394.

Nicoletti, N. F., Erig, T. C., Zanin, R. F., Pereira, T. C., Bogo, M. R., Campos, M. M., \& Morrone, F. B. (2014). Mechanisms involved in kinin-induced glioma cells proliferation: the role of ERK1/2 and PI3K/Akt pathways. Journal of Neuro-Oncology, 120(2), 235-244. http://dx.doi.org/10.1007/s11060-014-1549-4. PMid:25056222.

Pearse, D. D., Bastidas, J., Izabel, S. S., \& Ghosh, M. (2018). Schwann cell transplantation subdues the pro-inflammatory innate immune cell response after spinal cord injury. International Journal of Molecular Sciences, 19(9), 2550. http://dx.doi.org/10.3390/ijms19092550. PMid:30154346.

Qu, B., He, J., Zeng, Z., Yang, H., Liu, Z., Cao, Z., Yu, H., Zhao, W., \& Pan, X. (2020). MiR-155 inhibition alleviates suppression of osteoblastic differentiation by high glucose and free fatty acids in human bone marrow stromal cells by upregulating SIRT1. Pflügers Archiv, 472(4), 473-480. http://dx.doi.org/10.1007/s00424-02002372-7. PMid:32248286.

Ramírez-Moya, J., Wert-Lamas, L., \& Santisteban, P. (2018). MicroRNA$146 \mathrm{~b}$ promotes PI3K/AKT pathway hyperactivation and thyroid cancer progression by targeting PTEN. Oncogene, 37(25), 3369-3383. http://dx.doi.org/10.1038/s41388-017-0088-9. PMid:29353884. 
Sempere, L. F., Keto, J., \& Fabbri, M. (2017). Exosomal MicroRNAs in breast cancer towards diagnostic and therapeutic applications. Cancers, 9(12), 71. http://dx.doi.org/10.3390/cancers9070071. PMid:28672799.

Vidal, A. R., Cansian, R. L., Mello, R. O., Kubota, E. H., Demiate, I. M., Zielinski, A. A. F., \& Dornelles, R. C. R. P. (2020). Effect of ultrasound on the functional and structural properties of hydrolysates of different bovine collagens. Food Science and Technology, 40(2), 346-353. http://dx.doi.org/10.1590/fst.00319.

Wahl, E. A., Schenck, T. L., Machens, H. G., \& Balmayor, E. R. (2016). VEGF released by deferoxamine preconditioned mesenchymal stem cells seeded on collagen-GAG substrates enhances neovascularization. Scientific Reports, 6(1), 36879. http://dx.doi.org/10.1038/srep36879. PMid:27830734.

Walker, C. L., Liu, N. K., \& Xu, X. M. (2013). PTEN/PI3K and MAPK signaling in protection and pathology following CNS injuries. Frontiers of Biology, 8(4), 421-433. http://dx.doi.org/10.1007/s11515013-1255-1. PMid:24348522.
Wang, W., Dong, J., Wang, M., Yao, S., Tian, X., Cui, X., Fu, S., \& Zhang, S. (2018). miR-148a-3p suppresses epithelial ovarian cancer progression primarily by targeting c-Met. Oncology Letters, 15(5), 6131-6136. http://dx.doi.org/10.3892/ol.2018.8110. PMid:29616095.

Wu, J., He, Y., Luo, Y., Zhang, L., Lin, H., Liu, X., Liu, B., Liang, C., Zhou, Y., \& Zhou, J. (2018). MiR-145-5p inhibits proliferation and inflammatory responses of RMC through regulating AKT/GSK pathway by targeting CXCL16. Journal of Cellular Physiology, 233(4), 3648-3659. http://dx.doi.org/10.1002/jcp.26228. PMid:29030988.

Xu, L., Liu, Y., Sun, Y., Li, H., Mi, W., \& Jiang, Y. (2018). Analgesic effects of TLR4/NF-kappaB signaling pathway inhibition on chronic neuropathic pain in rats following chronic constriction injury of the sciatic nerve. Biomedicine and Pharmacotherapy, 107, 526-533. http://dx.doi.org/10.1016/j.biopha.2018.07.116. PMid:30114636.

Zhu, R., Bi, L., Kong, H., Xie, W., Hong, Y., \& Wang, H. (2015). Ruscogenin exerts beneficial effects on monocrotaline-induced pulmonary hypertension by inhibiting NF-kappaB expression. International Journal of Clinical and Experimental Pathology, 8(10), 12169-12176. PMid:26722401. 